\title{
Einstein-Cartan Portal to Dark Matter
}

\author{
Mikhail Shaposhnikov@ ${ }^{*}$ Andrey Shkerin $\odot,{ }^{\dagger}$ Inar Timiryasov $\odot,{ }^{\ddagger}$ and Sebastian Zell ${ }^{\S}$ \\ Institute of Physics, Laboratory for Particle Physics and Cosmology, École Polytechnique Fédérale de Lausanne, \\ CH-1015 Lausanne, Switzerland
}

(Received 2 September 2020; revised 14 January 2021; accepted 22 March 2021; published 22 April 2021)

\begin{abstract}
It is well known since the works of Utiyama and Kibble that the gravitational force can be obtained by gauging the Poincaré group, which puts gravity on the same footing as the standard model fields. The resulting theory-Einstein-Cartan gravity-inevitably contains four-fermion and scalar-fermion interactions that originate from torsion associated with spin degrees of freedom. We show that these interactions lead to a novel mechanism for producing singlet fermions in the early Universe. These fermions can play the role of dark matter particles. The mechanism is operative in a large range of dark matter particle masses: from a few $\mathrm{keV}$ up to $\sim 10^{8} \mathrm{GeV}$. We discuss potential observational consequences of keV-scale dark matter produced this way, in particular for right-handed neutrinos. We conclude that a determination of the primordial dark matter momentum distribution might be able to shed light on the gravity-induced fermionic interactions.
\end{abstract}

DOI: $10.1103 /$ PhysRevLett.126.161301

Introduction.-Gravity is a universal force, and it inevitably reveals some properties of yet-to-be-discovered constituents of nature: dark matter (DM) and dark energy (see, e.g., [1-3] for reviews). Indeed, all evidence for DM in galaxies and galaxy clusters and at large scales is due to the gravitational interaction. Thus, we know well how DM gravitates but little about what it is. The list of DM particle candidates includes, e.g., weakly interacting massive particles (see [4-8] for important early contributions), sterile neutrinos [9], axions [10-12] and axionlike particles [13]. Regardless of the nature of DM, any model of it should explain how it was produced in the early Universe and how its abundance is maintained.

In this Letter, we argue that gravity might be able to tell us not only about the distribution of DM in the Universe but also about the mechanism of its production. The two key ingredients of the framework allowing for such a statement are the Einstein-Cartan (EC) formulation of gravity [14,15] and the assumption about the fermion nature of a DM candidate. Regarding the second one, we take a DM particle to be a Dirac or Majorana fermion that is a singlet under the gauge group of the standard model (SM).

We begin with a brief discussion of EC gravity (see, e.g., $[16,17]$ for reviews). There is no doubt that below the Planck scale, general relativity (GR) provides an elegant and accurate description of gravity. Nevertheless, this leaves

Published by the American Physical Society under the terms of the Creative Commons Attribution 4.0 International license. Further distribution of this work must maintain attribution to the author(s) and the published article's title, journal citation, and DOI. Funded by SCOAP . still unanswered the question about which formulation of GR one should employ. An important alternative to the most widely used metric gravity is the EC formulation. In this theory, the role of fundamental fields is played by the tetrad and the spin connection, in terms of which the metric and the Christoffel symbols are introduced. The latter are, in general, not symmetric in the lower indices; hence, EC gravity contains torsion. Still, the number of propagating degrees of freedom - two of the graviton-is the same as in the metric formulation. A conceptual advantage of EC gravity is that it can be viewed as a gauge theory of the Poincaré group, thus allowing for a similar treatment of all fundamental forces. (Moreover, we note that in EC gravity there is no need for the Gibbons-Hawking-York boundary term [18,19] for the variational problem to be well posed).

In the absence of matter, the EC and metric formulations of gravity are equivalent. This changes once scalar fields coupled nonminimally to the Ricci scalar are introduced. The resulting theory is then equivalent to the Palatini formulation of gravity [20,21] (see also [22]). In this version of gravity, the metric and the Christoffel symbols are viewed as independent fundamental variables, and the latter are assumed to be symmetric in the lower indices. Another way to break the equivalence of the EC and metric formulations is to take into account fermions since the latter source torsion. The theory admits an exact solution for the torsionful part of the connection. Plugging this solution back in the action, one arrives at an equivalent theory in the metric formulation. The difference of the two theories of gravity is then manifested in the appearance of dimensionsix terms representing an interaction of fermionic axial currents [15,23] [24]. Their strength is fixed and suppressed by $1 / M_{P}^{2}$. 
In the statements thus far, we have considered in EC gravity the same action as in the metric theory. Because of the presence of torsion, however, one can form more terms of mass dimension not bigger than four than in the metric case. Specifically, the fermion kinetic terms can be generalized by introducing nonminimal fermion couplings [25-28]. In an equivalent metric theory, these couplings lead to vector-vector, axial-vector, and axial-axial fermion current interactions. Furthermore, the gravitational action of the EC theory can be extended by adding the Holst term [29-32], which modifies the four-fermion interaction. Note that the additional terms come with a priori unknown coupling constants and that the strength of current-current interactions depends on these couplings.

This Letter uses the results of [33], where a theory of scalar and fermion fields coupled to gravity was studied. There, we included additional terms of mass dimension not bigger than four that are specific to the EC formulation and derived the equivalent metric theory. When applied to cosmology and experiment, the scalar field can be associated with the SM Higgs field and the fermions with the SM quarks and leptons, as well as, possibly, additional species such as right-handed neutrinos. The phenomenology of the scalar-gravity part of the EC theory has already been investigated in [34]. There, we considered the Higgs field as an inflaton. Our study was motivated by the wellknown fact that the Higgs field can be responsible for inflation provided that it couples nonminimally to the Ricci scalar [35]. The models of Higgs inflation in the metric [35] and Palatini [36] formulations of gravity find their natural generalization within the EC theory [34] (see also [37]).

The goal of this Letter is to study the phenomenology of the fermionic sector of the EC theory. Namely, we show that the four-fermion interaction originating from EC gravity can be responsible for DM production. We compute the abundance and the spectrum of produced (Dirac or Majorana) particles and show that the right amount of DM can be generated for a wide range of fermion masses. We also discuss an interesting case of warm DM where the primordial momentum distribution characteristic for $\mathrm{EC}$ gravity can potentially be observable.

Einstein-Cartan gravity and fermions.-In this Letter, we focus on the fermion-gravity part of the general theory studied in [33]. To simplify the presentation, we only keep the Einstein-Hilbert term and the nonminimal couplings of fermions in the action. We comment on the inclusion of other terms later (see also Appendix A of the Supplemental Material [38]). Then, for each (Dirac or Majorana) fermion species $\Psi$, the relevant part of the action reads as follows [25-28] [39]:

$$
\begin{aligned}
\mathcal{L}= & \frac{1}{2} M_{P}^{2} R+\frac{i}{2} \bar{\Psi}\left(1-i \alpha-i \beta \gamma^{5}\right) \gamma^{\mu} D_{\mu} \Psi \\
& -\frac{i}{2} \overline{D_{\mu} \Psi}\left(1+i \alpha+i \beta \gamma^{5}\right) \gamma^{\mu} \Psi,
\end{aligned}
$$

where $M_{P}=2.435 \times 10^{18} \mathrm{GeV}$ is the Planck mass and $D_{\mu}$ is the covariant derivative containing the connection field. The real couplings $\alpha, \beta$ are chosen to be the same for all generations of fermions, which implies the universality of gravity in the fermionic sector. Allowing for the couplings to depend on a generation index yields qualitatively the same results. In metric gravity, the nonminimal terms sum up to a total derivative, but in the torsionful case they contribute to the dynamics of the theory.

The theory, Eq. (1), can be resolved for torsion explicitly $[15,23,25-28,33]$. Upon substituting the solution for torsion back to the action, one obtains an equivalent metric theory with extra higher-dimensional fermion interaction terms. It reads as follows:

$$
\mathcal{L}_{4 f}=\frac{-3 \alpha^{2}}{16 M_{P}^{2}} V^{\mu} V_{\mu}-\frac{3 \alpha \beta}{8 M_{P}^{2}} V^{\mu} A_{\mu}+\frac{3-3 \beta^{2}}{16 M_{P}^{2}} A^{\mu} A_{\mu},
$$

where $V^{\mu}=\bar{N} \gamma^{\mu} N+\sum_{X} \bar{X} \gamma^{\mu} X$ is the vector fermion current and $A^{\mu}$ is the analogous axial current (with $\gamma^{\mu}$ replaced by $\gamma^{5} \gamma^{\mu}$ ). The sum is performed over all SM fermion species $X$. For convenience, we wrote separately the terms containing $N$, which plays the role of DM and can be a Dirac or right-handed Majorana fermion. The interaction, Eq. (2), vanishes only if $\alpha=0, \beta= \pm 1$, and in what follows we do not consider this particular choice of the couplings.

Adding the Holst term to Eq. (1) modifies the couplings in Eq. (2). Furthermore, including a scalar field coupled nonminimally to gravity results in additional scalar-fermion interactions. Below we focus on the four-fermion interaction, Eq. (2), whose contribution to the DM production dominates for small masses of $N$. We discuss the general case in Appendix A of the Supplemental Material [38], which includes Refs. [40,41].

Thermal production of singlet fermions.-The fourfermion interaction, Eq. (2), opens up the production channel of $N$ particles through the annihilation of the SM fermions $X$ via the reaction $X+\bar{X} \rightarrow N+\bar{N}$ [42]. The kinetic equation corresponding to this reaction takes the form

$$
\left(\frac{\partial}{\partial t}-H q_{i} \frac{\partial}{\partial q_{i}}\right) f_{N}(t, \vec{q})=R(\vec{q}, T),
$$

where $f_{N}$ is the phase-space density of $N, H$ is the Hubble rate, and $R$ is the collision integral, also referred to as a production rate. In an isotropic background, both $f_{N}$ and $R$ depend only on the absolute value of the spatial momentum $|q| \equiv|\vec{q}|$.

In what follows, we assume that all SM particles, including fermions, are in thermal equilibrium at the moment of DM production. To check the validity of this assumption, one would need a careful examination of the dynamics of bosonic and fermionic SM species at and after 
preheating, which goes beyond the scope of this Letter. We expect, however, that deviations from thermality do not change our results qualitatively.

As long as the concentration of $N$ remains small and we can neglect the inverse processes, the collision integral in Eq. (3) reads

$$
R=\frac{1}{2|q|} \sum_{X} \int \mathrm{d} \Pi\left|\overline{\mathcal{M}}_{X}\right|^{2} f_{X}\left(p_{1}\right) f_{\bar{X}}\left(p_{2}\right),
$$

where the sum runs over the SM species (24 left- and 21 right-handed fermions), $\overline{\mathcal{M}}_{X}$ is the amplitude of the process summed over all spinor indices, and $f_{X}$ is the distribution of $X$, which we assume to be the Fermi-Dirac one. The differential $\mathrm{d} \Pi$ is the phase-space volume element accounting for energy conservation [see Appendix B of the Supplemental Material [38] for the definition of $\mathrm{d} \Pi$, as well as details on the derivations of the subsequent Eqs. (5)-(9)]. The typical values of the momenta in Eq. (4) are large compared to the mass of $N$, so we neglect all masses when computing $\overline{\mathcal{M}}_{X}$. Introducing the dimensionless variable $y=E / T$, where $T$ is the temperature of the cosmic plasma and $E=|q|$, we arrive at

$$
R(E, T)=T^{5} \frac{C_{f}(\alpha, \beta)}{M_{P}^{4}} r(y) .
$$

Here, $C_{f}(\alpha, \beta)$ is a combination of the nonminimal fermion couplings whose precise form depends on whether $N$ is Majorana $(f=M)$ or Dirac $(f=D)$ :

$C_{M}=\frac{9}{4}\left(24 a^{4}+21 b_{+}^{4}\right), \quad C_{D}=\frac{9}{4}\left(45 a^{4}+21 b_{+}^{4}+24 b_{-}^{4}\right)$,

where $a^{2}=1+\alpha^{2}-\beta^{2}, b_{ \pm}^{2}=1-(\alpha \pm \beta)^{2}$. Next, $r(y)$ is a function that we compute numerically (following [44]). It is accurately approximated by

$$
r(y) \simeq \frac{1}{24 \pi^{3}} y f_{X}
$$

(This expression is exact if, instead of the Fermi-Dirac distribution, one uses the Boltzmann distribution for $f_{X}$.) Eq. (3) can now be easily integrated, leading to

$$
f_{N}(y)=\frac{C_{f} T_{\mathrm{prod}}^{3} M_{0}\left(T_{\mathrm{prod}}\right)}{3 M_{P}^{4}} r(y),
$$

where $T_{\text {prod }}$ is the temperature at which the DM production begins, $M_{0}(T)=M_{P} \sqrt{\left[90 / \pi^{2} g_{\text {eff }}(T)\right]}$, and $g_{\text {eff }}\left(T_{\text {prod }}\right)=$ 106.75 is the number of effectively massless degrees of freedom at high temperature. Plugging in the numbers, we obtain for the abundance of $N$ particles [45]

$$
\frac{\Omega_{N}}{\Omega_{\mathrm{DM}}} \simeq 3.6 \times 10^{-2} C_{f}\left(\frac{M_{N}}{10 \mathrm{keV}}\right)\left(\frac{T_{\text {prod }}}{M_{P}}\right)^{3},
$$

where $\Omega_{\mathrm{DM}}$ is the observed DM abundance and the coefficient $C_{f}$ is defined in Eq. (6). Equation (9) shows that, depending on the value of $C_{f}$, the right amount of DM can be generated in a broad range of fermion masses $M_{N}$.

To proceed further, we need an estimate for the production temperature. We obtain it within the framework of Higgs inflation in the Palatini formulation of gravity $[36,46]$. In this model, preheating is almost instantaneous [47], and one can take $T_{\text {prod }} \sim T_{\text {reh }}$ where

$$
T_{\text {reh }} \simeq\left(\frac{15 \lambda}{2 \pi^{2} g_{\text {eff }}}\right)^{1 / 4} \frac{M_{P}}{\sqrt{\xi}}
$$

is the preheating temperature, $\lambda$ is the Higgs field selfcoupling, and $\xi$ is the nonminimal coupling of the Higgs field to the Ricci scalar. Both $\lambda$ and $\xi$ are taken at a high energy scale. Using $\xi=10^{7}$ and $\lambda=10^{-3}$ [48], we obtain $T_{\text {prod }} \simeq 4 \times 10^{13} \mathrm{GeV}$.

Now we can investigate two particularly interesting cases. The first one is the limit of vanishing nonminimal couplings, $\alpha=\beta=0$. Then, from Eq. (9) we obtain that $\Omega_{N} \simeq \Omega_{\mathrm{DM}}$ if $M_{N} \simeq 6 \times 10^{8} \mathrm{GeV}$ for the Majorana fermion and $M_{N} \simeq 3 \times 10^{8} \mathrm{GeV}$ for the Dirac fermion. We conclude that heavy fermion DM can be produced in EC gravity even if the action of the EC theory is identical to that of the metric theory. (Such a heavy DM can also be produced due to graviton exchange between fermionic currents.) Interestingly, the given bounds on $M_{N}$ are close to the bound $M_{N} \lesssim 10^{9} \mathrm{GeV}$ above which $N$ particles are overproduced due to the varying geometry at the radiationdominated stage of the Universe [49-51].

The second case corresponds to setting $\alpha \sim \beta \sim \sqrt{\xi}$. With this choice, the scale of suppression of the interaction, Eq. (2), coincides with the inflationary cutoff scale which in Palatini Higgs inflation is of the order of $M_{P} / \sqrt{\xi}$ [52]. For both the Majorana and Dirac cases, Eq. (9) becomes

$$
\frac{\Omega_{N}}{\Omega_{\mathrm{DM}}} \simeq 1.4 \frac{\sqrt{\xi} \lambda^{3 / 4}}{g_{\mathrm{eff}}^{3 / 4}} \frac{(\alpha+\beta)^{4}}{\xi^{2}}\left(\frac{M_{N}}{10 \mathrm{keV}}\right)\left(\frac{T_{\text {prod }}}{T_{\text {reh }}}\right)^{3} .
$$

Thus, the right amount of DM is generated for a $\mathrm{keV}$ scale $M_{N}$.

Einstein-Cartan portal to warm dark matter.-Let us discuss in more detail the second choice of the nonminimal couplings. Then, $N$ is an example of warm DM, and its free-streaming length affects structure formation. Since DM is produced at very high temperatures, its spectrum is redshifted. Consequently, the average momentum is only $\simeq 0.61$ of the equilibrium momentum at $T=1 \mathrm{MeV}$. Depending on the history of reionization, such colder DM candidates can provide a better fit to the Lyman- $\alpha$ data than pure cold DM $[53,54]$. 


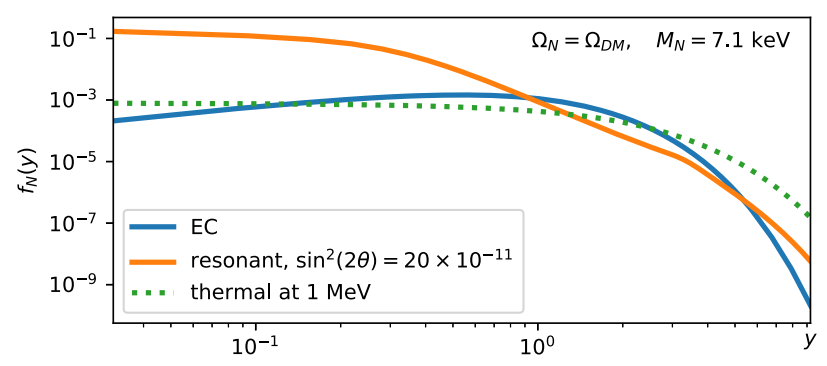

FIG. 1. The DM distribution function at $T=1 \mathrm{MeV}$. The normalization is such that $\Omega_{N}=\Omega_{\mathrm{DM}}$ for the benchmark mass $M_{N}=7.1 \mathrm{keV}$ used in [55]. The blue curve shows the redshifted spectrum, Eq. (8), whereas the orange one shows the spectrum of a resonantly produced sterile neutrino [55]. For comparison, we also show the thermal spectrum (the green dotted line).

In Fig. 1, we show the spectrum $f_{N}$ of DM produced in EC gravity. For comparison, we also show the spectrum of a resonantly produced sterile neutrino taken from [55] [56] and the thermal spectrum at $1 \mathrm{MeV}$. We see that the DM momentum distribution, Eq. (8), has a unique form and differs from both nonresonantly $[9,57]$ and resonantly produced sterile neutrinos [55,58-61]. This potentially allows one to distinguish DM produced via the EC portal from other DM candidates. We conclude that, at least for certain values of $M_{N}$, the distribution of DM in the Universe can bear the information about the gravity-induced fermionic interactions.

LMSM. - The lack of a DM candidate is a famous shortcoming of the SM, but it is not the only one. SM also cannot explain neutrino oscillations or the baryon asymmetry of the Universe. A possible way to address these three issues at once is the neutrino minimal standard model ( $\nu$ MSM), which extends the particle content of the SM by three right-handed neutrinos $N_{1,2,3}$ [62,63]. One of them, $N_{1}$, can be the DM candidate. A lower bound on its mass, $M_{N_{1}} \gtrsim 1 \mathrm{keV}$, comes from small-scale structures in the matter power spectrum, as inferred, e.g., from Lyman- $\alpha$ measurements. The mixing of $N_{1}$ with active neutrinos is bounded from above by x-ray constraints. The other two right-handed neutrinos, $N_{2,3}$, have nearly degenerate $\mathrm{GeV}$ scale masses and are responsible for generating the baryon asymmetry. Moreover, the parameters of the model can be chosen such that the observed pattern of neutrino oscillations is obtained (for reviews see $[64,65]$ ).

So far, the production of the DM sterile neutrino was associated with the mixing of $N_{1}$ with ordinary neutrinos and typical temperatures $100-300 \mathrm{MeV}$ [9,58]. The socalled nonresonant production of $N_{1}$ [9] has already been excluded by $\mathrm{x}$-ray searches of radiatively decaying DM [65]. The resonant production of a DM sterile neutrino [58] requires large lepton asymmetries that can be produced in interactions of $N_{2,3}$ [66]. It can be successful, albeit a finetuning of parameters is required [67-69]. For resonantly produced DM, the nonobservation of x-ray decays of DM in galactic halos leads to an upper bound on the mass
$M_{N_{1}} \lesssim 50 \mathrm{keV}$ [65], thereby constraining $M_{N_{1}}$ in a quite narrow range.

The present work supplies the $\nu$ MSM with a different mechanism to produce the $N_{1}$ particles. Sterile neutrino DM produced this way may be absolutely stable and therefore is not subject to any $\mathrm{x}$-ray constraints. This opens a new interesting mass window up to $M_{N_{1}} \sim 10^{8} \mathrm{GeV}$. The prediction of the $\iota \mathrm{MSM}$ that the lightest active neutrino is effectively massless remains in force also for large values of $M_{N_{1}}$ (see Appendix C of the Supplemental Material [38], which includes Refs. [70-72]). (Note that this prediction can potentially be tested by the Euclid space mission [73].)

It is very intriguing that if the perturbative cutoff is universal for both the fermion and scalar-gravity sectors of the EC theory $\left(\sim M_{P} / \sqrt{\xi}\right)$, then the mass of the DM sterile neutrino is required to be in the $\mathrm{keV}$ range. This is the domain where the warm nature of the DM particle is most visible and in which the most intensive searches of the radiatively decaying DM are being carried out.

We note that Majorana fermions $N_{2,3}$ will be also produced by the same mechanism. Their number density is the same as that of $N_{1}, n_{2,3} \simeq n_{1}$, and the latter is fixed by the DM abundance $n_{1} \simeq 10^{-2} n_{\text {eq }}\left(10 \mathrm{keV} / M_{N_{1}}\right)$. However, this density is too small to affect the analysis of the $\nu \mathrm{MSM}$ leptogenesis carried out previously (e.g., [74]) (see Appendix D of the Supplemental Material [38]).

Discussion and outlook.-Once gravity is coupled to matter such as fermions or a nonminimally coupled scalar field, its different formulations are no longer equivalent. As long as the theories are consistent, only observations can help us to distinguish between them. In this Letter, we have shown that the properties of fermionic dark matter may be able to discern the EC theory of gravity from the most commonly used metric formulation. In particular, the universal dimension-six interactions of fermionic currents in EC theory can cause the production of the observed amount of dark matter for a wide range of fermion masses. Moreover, they lead to a characteristic momentum distribution of dark matter, which can serve to confirm or exclude our proposed production mechanism.

On the one hand, these findings are relevant for any extension of the SM by sufficiently heavy fermions. On the other hand, an exciting unified picture of gravity and the $\mathrm{SM}$ emerges. It relies on the EC formulation of gravity and the extension of SM by three right-handed neutrinos, i.e., the $\nu$ MSM $[62,63]$. When nonminimally coupled to the Ricci scalar, the Higgs field can assume the role of the inflaton [35]. As discussed in [34], the resulting inflationary scenario generalizes the model of Palatini Higgs inflation [36] and is fully compatible with observations. On its own, the $\nu \mathrm{MSM}$ is able to explain neutrino oscillations and baryogenesis and provides a dark matter candidate in the form of a right-handed neutrino. The result of the present work is that the EC formulation of gravity can lead to the production of this sterile neutrino in an amount that 
matches the observed abundance of dark matter. Finally, we remark that the Palatini formulation of gravity and, by generalization, EC gravity are convenient for addressing the question of the big difference between the electroweak and the Planck scales [75] (see also [76-79]).

We thank Alexey Boyarsky and Oleg Ruchayskiy for useful discussions. The work was supported by ERC-AdG2015 Grant No. 694896 and by the Swiss National Science Foundation Excellence Grant No. 200020B_182864.

*Corresponding author.

mikhail.shaposhnikov@epfl.ch

${ }^{\dagger}$ Corresponding author.

andrey.shkerin@epfl.ch

${ }^{*}$ Corresponding author.

inar.timiryasov@epfl.ch

${ }^{\S}$ Corresponding author.

sebastian.zell@epfl.ch

[1] J. L. Feng, Dark matter candidates from particle physics and methods of detection, Annu. Rev. Astron. Astrophys. 48, 495 (2010).

[2] G. Bertone and D. Hooper, History of dark matter, Rev. Mod. Phys. 90, 045002 (2018).

[3] K. Arun, S. B. Gudennavar, and C. Sivaram, Dark matter, dark energy, and alternate models: A review, Adv. Space Res. 60, 166 (2017).

[4] H. Pagels and J. R. Primack, Supersymmetry, Cosmology and New TeV Physics, Phys. Rev. Lett. 48, 223 (1982).

[5] S. Weinberg, Upper Bound on Gauge Fermion Masses, Phys. Rev. Lett. 50, 387 (1983).

[6] H. Goldberg, Constraint on the Photino Mass from Cosmology, Phys. Rev. Lett. 50, 1419 (1983); Erratum, Phys. Rev. Lett. 103, 099905 (2009).

[7] J. R. Ellis, J. S. Hagelin, D. V. Nanopoulos, and M. Srednicki, Search for supersymmetry at the anti-p p collider, Phys. Lett. 127B, 233 (1983).

[8] J. R. Ellis, J. S. Hagelin, D. V. Nanopoulos, K. A. Olive, and M. Srednicki, Supersymmetric relics from the big bang, Nucl. Phys. B238, 453 (1984).

[9] S. Dodelson and L. M. Widrow, Sterile-Neutrinos as Dark Matter, Phys. Rev. Lett. 72, 17 (1994).

[10] J. Preskill, M. B. Wise, and F. Wilczek, Cosmology of the invisible axion, Phys. Lett. B 120, 127 (1983).

[11] L. F. Abbott and P. Sikivie, A cosmological bound on the invisible axion, Phys. Lett. 120B, 133 (1983).

[12] M. Dine and W. Fischler, The not so harmless axion, Phys. Lett. 120B, 137 (1983).

[13] W. Hu, R. Barkana, and A. Gruzinov, Cold and Fuzzy Dark Matter, Phys. Rev. Lett. 85, 1158 (2000).

[14] R. Utiyama, Invariant theoretical interpretation of interaction, Phys. Rev. 101, 1597 (1956).

[15] T. W. B. Kibble, Lorentz invariance and the gravitational field, J. Math. Phys. (N.Y.) 2, 212 (1961).

[16] F. W. Hehl, P. Von Der Heyde, G. D. Kerlick, and J. M. Nester, General relativity with spin and torsion: Foundations and prospects, Rev. Mod. Phys. 48, 393 (1976).
[17] I.L. Shapiro, Physical aspects of the space-time torsion, Phys. Rep. 357, 113 (2002).

[18] J. W. York, Jr., Role of Conformal Three Geometry in the Dynamics of Gravitation, Phys. Rev. Lett. 28, 1082 (1972).

[19] G. W. Gibbons and S. W. Hawking, Action integrals and partition functions in quantum gravity, Phys. Rev. D 15, 2752 (1977).

[20] A. Palatini, Deduzione invariantiva delle equazioni gravitazionali dal principio di hamilton, Rend. Circ. Mat. Palermo 43, 203 (1919).

[21] A. Einstein, Einheitliche Feldtheorie von Gravitation und Elektrizität, Sitzungsber. Preuss. Akad. Wiss. Phys. Math. 22, 414 (1925).

[22] M. Ferraris, M. Francaviglia, and C. Reina, Variational formulation of general relativity from 1915 to 1925 "Palatini's method" discovered by einstein in 1925, Gen. Relativ. Gravit. 14, 243 (1982).

[23] V. I. Rodichev, Twisted space and nonlinear field equations, JETP Lett. 13, 1029 (1961), http://www.jetp.ac.ru/cgi-bin/ dn/e_013_05_1029.pdf.

[24] Of course, one could have started from the beginning in a metric theory with additional higher-dimensional operators. In this case, however, a consistent effective field theory approach would dictate that all possible higher-dimensional interactions (consistent with relevant symmetries) be taken into account.

[25] L. Freidel, D. Minic, and T. Takeuchi, Quantum gravity, torsion, parity violation and all that, Phys. Rev. D 72, 104002 (2005).

[26] S. Alexandrov, Immirzi parameter and fermions with nonminimal coupling, Classical Quantum Gravity 25, 145012 (2008).

[27] D. Diakonov, A. G. Tumanov, and A. A. Vladimirov, Lowenergy general relativity with torsion: A systematic derivative expansion, Phys. Rev. D 84, 124042 (2011).

[28] J. Magueijo, T. G. Zlosnik, and T. W. B. Kibble, Cosmology with a spin, Phys. Rev. D 87, 063504 (2013).

[29] R. Hojman, C. Mukku, and W. A. Sayed, Parity violation in metric torsion theories of gravitation, Phys. Rev. D 22, 1915 (1980).

[30] P.C. Nelson, Gravity with propagating pseudoscalar torsion, Phys. Lett. 79A, 285 (1980).

[31] L. Castellani, R. D'Auria, and P. Frè, Supergravity and Superstrings: A Geometric Perspective. Vol. 1: Mathematical Foundations (World Scientific, Singapore, 1991).

[32] S. Holst, Barbero's Hamiltonian derived from a generalized Hilbert-Palatini action, Phys. Rev. D 53, 5966 (1996).

[33] M. Shaposhnikov, A. Shkerin, I. Timiryasov, and S. Zell, Einstein-Cartan gravity, matter, and scale-invariant generalization, J. High Energy Phys. 10 (2020) 177.

[34] M. Shaposhnikov, A. Shkerin, I. Timiryasov, and S. Zell, Higgs inflation in Einstein-Cartan gravity, J. Cosmol. Astropart. Phys. 02 (2021) 008.

[35] F. L. Bezrukov and M. Shaposhnikov, The standard model Higgs boson as the inflaton, Phys. Lett. B 659, 703 (2008).

[36] F. Bauer and D. A. Demir, Inflation with non-minimal coupling: Metric versus Palatini formulations, Phys. Lett. B 665, 222 (2008). 
[37] M. Långvik, J.-M. Ojanperä, S. Raatikainen, and S. Rasanen, Higgs inflation with the Holst and the Nieh-Yan term, arXiv:2007.12595 [Phys. Rev. D (to be published)].

[38] See Supplemental Material at http://link.aps.org/supplemental/ 10.1103/PhysRevLett.126.161301 for the general form of action, the details of the DM abundance calculation, the discussion of the mass hierarchy of active neutrinos, and the discussion of the effect on leptogenesis.

[39] We work in natural units $\hbar=c=1$ and use the metric signature $(-1,+1,+1,+1)$. The matrix $\gamma^{5}$ is defined as $\gamma^{5}=-i \gamma^{0} \gamma^{1} \gamma^{2} \gamma^{3}$

[40] G. Immirzi, Quantum gravity and Regge calculus, Nucl. Phys. B, Proc. Suppl. 57, 65 (1997).

[41] G. Immirzi, Real and complex connections for canonical gravity, Classical Quantum Gravity 14, L177 (1997).

[42] The production of singlet fermions due to some higherdimensional operators was considered in [43]. However, the four-fermion interaction that appears in EC gravity was not accounted for.

[43] F. Bezrukov, D. Gorbunov, and M. Shaposhnikov, Late and early time phenomenology of Higgs-dependent cutoff, J. Cosmol. Astropart. Phys. 10 (2011) 001.

[44] T. Asaka, M. Laine, and M. Shaposhnikov, Lightest sterile neutrino abundance within the numsm, J. High Energy Phys. 01 (2007) 091; Erratum, J. High Energy Phys. 02 (2015) 028.

[45] In deriving this result, we assume that all other possible interactions of $N$ particles with particles of the SM are not essential for the DM production.

[46] T. Tenkanen, Tracing the high energy theory of gravity: An introduction to Palatini inflation, Gen. Relativ. Gravit. 52, 33 (2020).

[47] J. Rubio and E. S. Tomberg, Preheating in Palatini Higgs inflation, J. Cosmol. Astropart. Phys. 04 (2019) 021.

[48] M. Shaposhnikov, A. Shkerin, and S. Zell, Quantum effects in Palatini Higgs inflation, J. Cosmol. Astropart. Phys. 07 (2020) 064.

[49] S. G. Mamaev, V. M. Mostepanenko, and A. A. Starobinsky, Particle creation from vacuum near an homogeneous isotropic singularity, JETP 43, 823 (1976), http://www.jetp.ac .ru/cgi-bin/e/index/e/43/5/p823?a=list.

[50] V. Kuzmin and I. Tkachev, Ultrahigh-energy cosmic rays, superheavy long living particles, and matter creation after inflation, JETP Lett. 68, 271 (1998).

[51] D. J. H. Chung, E. W. Kolb, and A. Riotto, Nonthermal Supermassive Dark Matter, Phys. Rev. Lett. 81, 4048 (1998).

[52] F. Bauer and D. A. Demir, Higgs-Palatini inflation and unitarity, Phys. Lett. B 698, 425 (2011).

[53] A. Garzilli, A. Magalich, T. Theuns, C. S. Frenk, C. Weniger, O. Ruchayskiy, and A. Boyarsky, The Lyman- $\alpha$ forest as a diagnostic of the nature of the dark matter, Mon. Not. R. Astron. Soc. 489, 3456 (2019).

[54] A. Garzilli, O. Ruchayskiy, A. Magalich, and A. Boyarsky, How warm is too warm? Towards robust Lyman- $\alpha$ forest bounds on warm dark matter, arXiv:1912.09397.

[55] J. Ghiglieri and M. Laine, Improved determination of sterile neutrino dark matter spectrum, J. High Energy Phys. 11 (2015) 171.

[56] This result depends on the assumptions about the lepton asymmetry and the values of Yukawa coupling. The curve in
Fig. 1 corresponds to the "case a," $\sin ^{2}(2 \theta)=20 \times 10^{-11}$ in terms of [55].

[57] A. D. Dolgov and S. H. Hansen, Massive sterile neutrinos as warm dark matter, Astropart. Phys. 16, 339 (2002).

[58] X.-D. Shi and G. M. Fuller, A New Dark Matter Candidate: Nonthermal Sterile Neutrinos, Phys. Rev. Lett. 82, 2832 (1999).

[59] M. Laine and M. Shaposhnikov, Sterile neutrino dark matter as a consequence of numsm-induced lepton asymmetry, J. Cosmol. Astropart. Phys. 06 (2008) 031.

[60] K. Abazajian, G. M. Fuller, and M. Patel, Sterile neutrino hot, warm, and cold dark matter, Phys. Rev. D 64, 023501 (2001).

[61] T. Venumadhav, F.-Y. Cyr-Racine, K. N. Abazajian, and C. M. Hirata, Sterile neutrino dark matter: Weak interactions in the strong coupling epoch, Phys. Rev. D 94, 043515 (2016).

[62] T. Asaka, S. Blanchet, and M. Shaposhnikov, The nuMSM, dark matter and neutrino masses, Phys. Lett. B 631, 151 (2005).

[63] T. Asaka and M. Shaposhnikov, The $\nu$ MSM, dark matter and baryon asymmetry of the universe, Phys. Lett. B 620, 17 (2005).

[64] A. Boyarsky, O. Ruchayskiy, and M. Shaposhnikov, The Role of sterile neutrinos in cosmology and astrophysics, Annu. Rev. Nucl. Part. Sci. 59, 191 (2009).

[65] A. Boyarsky, M. Drewes, T. Lasserre, S. Mertens, and O. Ruchayskiy, Sterile neutrino dark matter, Prog. Part. Nucl. Phys. 104, 1 (2019).

[66] M. Shaposhnikov, The nuMSM, leptonic asymmetries, and properties of singlet fermions, J. High Energy Phys. 08 (2008) 008.

[67] L. Canetti, M. Drewes, and M. Shaposhnikov, Sterile Neutrinos as the Origin of Dark and Baryonic Matter, Phys. Rev. Lett. 110, 061801 (2013).

[68] L. Canetti, M. Drewes, T. Frossard, and M. Shaposhnikov, Dark matter, baryogenesis and neutrino oscillations from right handed neutrinos, Phys. Rev. D 87, 093006 (2013).

[69] J. Ghiglieri and M. Laine, Sterile neutrino dark matter via coinciding resonances, J. Cosmol. Astropart. Phys. 07 (2020) 012.

[70] A. Boyarsky, A. Neronov, O. Ruchayskiy, and M. Shaposhnikov, The masses of active neutrinos in the nuMSM from X-ray astronomy, JETP Lett. 83, 133 (2006).

[71] P. B. Pal and L. Wolfenstein, Radiative decays of massive neutrinos, Phys. Rev. D 25, 766 (1982).

[72] V. D. Barger, R. J. N. Phillips, and S. Sarkar, Remarks on the KARMEN anomaly, Phys. Lett. B 352, 365 (1995); Erratum, Phys. Lett. B 356, 617 (1995).

[73] B. Audren, J. Lesgourgues, S. Bird, M. G. Haehnelt, and M. Viel, Neutrino masses and cosmological parameters from a Euclid-like survey: Markov Chain Monte Carlo forecasts including theoretical errors, J. Cosmol. Astropart. Phys. 01 (2013) 026.

[74] J. Klarić, M. Shaposhnikov, and I. Timiryasov, Uniting lowscale leptogeneses, arXiv:2008.13771.

[75] M. Shaposhnikov, A. Shkerin, and S. Zell, Standard Model meets gravity: Electroweak symmetry breaking and inflation, Phys. Rev. D 103, 033006 (2021).

[76] M. Shaposhnikov and A. Shkerin, Conformal symmetry: Towards the link between the Fermi and the Planck scales, Phys. Lett. B 783, 253 (2018). 
[77] M. Shaposhnikov and A. Shkerin, Gravity, scale invariance and the hierarchy problem, J. High Energy Phys. 10 (2018) 024.

[78] A. Shkerin, Dilaton-assisted generation of the Fermi scale from the Planck scale, Phys. Rev. D 99, 115018 (2019).
[79] G. K. Karananas, M. Michel, and J. Rubio, One residue to rule them all: Electroweak symmetry breaking, inflation and field-space geometry, Phys. Lett. B 811, 135876 (2020). 